BULLETIN OF THE

AMERICAN MATHEMATICAL SOCIETY

Volume 79, Number 1, January 1973

\title{
MANIFOLDS AS OPEN BOOKS
}

\author{
BY H. E. WINKELNKEMPER ${ }^{1}$
}

Communicated by Michael Atiyah, June 5, 1972

Introduction. We prove that all closed, simply-connected, differentiable (or p.l.) manifolds of dimension $>6$ and index $\tau=0$ decompose in a certain way: as "open books", a decomposition analogous to the classical Lefschetz decomposition of nonsingular algebraic varieties. The condition $\tau=0$ is also necessary for a manifold to be an open book, and so, in particular, we have found a simple, intrinsic, geometric equivalent of it. For any orientable 3-manifold, this decomposition had been given by Alexander in 1923, using properties of branched coverings, which do not seem to generalize to higher dimensions. The proof of our theorem is not difficult and is a natural consequence of decomposing manifolds à la Heegaard, first accomplished for a large class of high-dimensional manifolds by Smale and completed by others [2], [3], [8]. ${ }^{2}$

We wish to thank W. D. Neumann, J. W. Wood and especially Professor D. Sullivan of M.I.T. for very helpful conversations.

1. Statement of the theorem. Let $V$ be a compact differentiable $(n-1)$ manifold with $\partial V \neq \varnothing$ and $h: V \rightarrow V$ a diffeomorphism, which restricts to the identity on $\partial V$; by forming the mapping torus $V_{h}$, which has $\partial V \times S^{1}$ as boundary, and identifying $(x, t) \sim\left(x, t^{\prime}\right)$ on $\partial V_{h}$ for each $x \in \partial V, t, t^{\prime} \in S^{1}$, we obtain a closed, differentiable $n$-manifold $M$, which, if we look at a piece of the image $N$ of $\partial V \times S^{1}$ under the identification map, looks like an open book (Figure 1).

The fibers of $V$ define the 'pages' and $N$, a closed codimension 2 submanifold is called the 'binding'. Every point $x \notin N$ lies on one and only one page and the boundary of each page coincides with $N$.

Definition. A closed manifold is an open book if it is diffeomorphic to one of those just obtained.

Hence an open book is represented by a page $V$ and a self-diffeomorphism $h: V \rightarrow V$, which restricts to the identity on $\partial V$. If $h_{*}: H_{*}(V, Z) \rightarrow$ $H_{*}(V, Z)$ is the identity, we say that the open book decomposition has no monodromy.

AMS (MOS) subject classifications (1970). Primary 57A15, 57D15; Secondary 57C20, 57D65, 57D80.

Key words and phrases. Open book decomposition, minimal handle decomposition, $h$-cobordism, pages, binding, monodromy.

${ }^{1}$ Supported in part by National Science Foundation grant GP-7952X3.

2 Also, J. P. Alexander (Ph.D. Thesis, University of California, Berkeley, 1971). 




Figure 1

EXAMPLES. (a) Fibered knots. These are open book decompositions of spheres, where the bindings are also spheres.

(b) Milnor's Fibration Theorem [4] gives many nontrivial open book decompositions of $S^{2 k+1}$, whose monodromy is interesting.

(c) Let $V$ be any compact manifold with $\partial V \neq \varnothing$; in $V \times I$ identify each interval $(x, t), x \in \partial V, t \in I$, to a point $\left(x, \frac{1}{2}\right)$ obtaining a manifold with boundary, $W$; let $N \subset \partial W$ be the image of $\partial V \times I$ under the identification, which divides $\partial W$ into $(\partial W)_{+}$and $(\partial W)_{-} ;$if $h:\left(\partial W,(\partial W)_{+},(\partial W)_{-}\right) \rightarrow$ $\left(\partial W,(\partial W)_{+},(\partial W)_{-}\right)$is a diffeomorphism of triples, then $W \cup_{h} W$ has an open book decomposition with binding $N$.
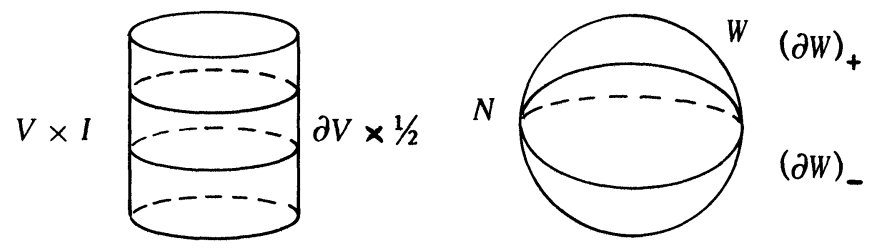

FIGURE 2

In 1923, Alexander [1] proved: Every orientable 3-manifold is an open book. We extend Alexander's theorem to dimensions $>6$.

OPEN BOOK THEOREM. Let $n>6$ and $M$ be a closed, simply-connected n-manifold.

Part 1. (a) If $n \not \equiv 0 \bmod 4, M$ is an open book.

(b) If $n \equiv 0 \bmod 4, M$ is an open book if and only if the index $\tau(M)=0$.

Furthermore, our pages and bindings will also be simply-connected and $H_{i}(V, Z)=0$ for $i>[n / 2]$. We now normalize the open book definition by requiring that $H_{i}(V, Z)=0$ for $i>[n / 2]$. 
Part 2. (a) For odd n, $M$ has an open book decomposition with no monodromy if and only if $H_{k}(M, Z)$ has no torsion $(n=2 k+1)$.

(b) If $n \equiv 2 \bmod 4, M$ always has an open book decomposition with no monodromy.

(c) If $n \equiv 0 \bmod 4$ and $M$ is of type I, $M$ has an open book decomposition with no monodromy if and only if $\tau(M)=0$.

(d) If $n \equiv 0 \bmod 4$ and $M$ is of type II, $M$ has an open book decomposition with no monodromy, if and only if $\tau(M)=0$ and the middle-dimensional $W u$ class $V_{n / 2}(M)=0$.

REMARK. Recall that $M$ is called of type II if all numbers in the diagonal of its intersection matrix, with respect to some basis of $H_{m}(M, Z) \bmod$ torsion, are even; of type I, if it is not of type II $(n=2 m)$.

2. Proof of part I for $n=2 k+1>5$. The proof of this case already illustrates all the ideas involved in proving our theorem. Following Smale [5, Theorem 8.1], fix a minimal handle decomposition of $M^{2 k+1}$ and let $W_{1}$ be constructed by all $i$-handles for $i \leqq k$ and $W_{2}$ with all such $i$-handles of the dual handle decomposition. Then $M=W_{1} \cup W_{2}$, $\partial W_{1}=\partial W_{2}=W_{1} \cap W_{2}=E$ (see Figure 3) and there exist $k$-dimensional subcomplexes $K_{l} \subset W_{l}(l=1,2)$ such that these inclusions are homotopy equivalences.

ASSERTION. There exists a $k$-complex $K \subset E \subset \partial W_{1}=\partial W_{2}$ such that both inclusions $K \subset W_{l}$ are homotopy equivalences.

Proof. Let $i_{l}: E \rightarrow K_{l}$ be the maps defined by $E \subset W_{l} \cong K_{l}$. Suppose we found a map $c: K_{1} \rightarrow E$ such that both $i_{1} c: K_{1} \rightarrow K_{1}$ and $i_{2} c: K_{1} \rightarrow K_{2}$ are homotopy equivalences. Then, since $\operatorname{dim} E=2 k$, if we put $c$ in general position, the only singularities will be transverse self-intersections of $k$-simplices of $K_{1}$. By a well known method, due to Stallings (embedding 'up to homotopy', see [6] for example), we can attach 2-disks to the image of $c$ to obtain a complex $K \subset E=\partial W_{1}$, which is homotopically equivalent to $K_{1}$; here the condition $n=2 k+1>5$ is used.

In order to find $c$ notice the following (all homology groups are taken over the integers and $l=1,2)$ :

(a) $H_{k}\left(K_{l}\right)$ are free and, since the $W_{l}$ are defined with respect to a minimal handlebody decomposition, by duality, they have the same number of generators.

(b) By duality, $H_{i}(W, E)=0$ for $i \leqq k$ and, by the relative Hurewicz theorem, $\Pi_{i}\left(W_{l}, E\right)=0$ for $i \leqq k$.

(c) By (b), the $i_{l *}: H_{i}(E) \rightarrow H_{i}\left(K_{l}\right)$ are isomorphisms for $i<k$ and epimorphisms for $i=k$.

(d) From (c) and the relative Hurewicz theorem, it follows that the $i_{l}$ are $(k-1)$-connected maps; i.e., if we consider them to be fiber maps (up to 
homotopy) with fibers $F_{l}$, then $\Pi_{i}\left(F_{l}\right)=0$ for $i<k$.

By (b), there exists a map $c^{\prime}: K_{1} \rightarrow E$ such that the diagram

$$
\begin{aligned}
& W_{1} \supset E \\
& \text { २॥ } / c^{\prime} \\
& K_{1}
\end{aligned}
$$

commutes up to homotopy and so $i_{1} c^{\prime}: K_{1} \rightarrow K_{1}$ induces isomorphisms in $H_{*}\left(K_{1}\right)$ and is therefore a homotopy equivalence; by composing with a homotopy equivalence we can suppose that $i_{1} c^{\prime}$ is homotopic to the identity $K_{1} \rightarrow K_{1}$ and so there exists at least one cross section of the fibering $F_{1} \rightarrow E \rightarrow K_{1}$. We wish to change this cross section, leaving it fixed on the $(k-1)$-skeleton of $K_{1}$, to a cross section $c: K_{1} \rightarrow E$ such that $i_{2} c: K_{1} \rightarrow K_{2}$ is also a homotopy equivalence. But the difference cocycle of two such cross sections lies in $H^{k}\left(K_{1}, \Pi_{k}(F)\right)$, where $K_{1}$ is $k$-dimensional and $\Pi_{i}(F)=0$ for $i<k$ (by $\left.(\mathrm{d})\right)$. Hence we are faced with a primary obstruction problem: If we can find any homomorphism $c_{*}: H_{k}\left(K_{1}\right) \rightarrow$ $H_{k}\left(K_{2}\right)$ such that $i_{1 *} c_{*}=$ identity and $i_{2 *} c_{*}$ is an isomorphism, then we can change our cross section $c^{\prime}$, without changing it on the $(k-1)$ skeleton of $K_{1}$, to a cross section $c: K_{1} \rightarrow E$ which induces $c_{*}$. By Whitehead's theorem, $i_{2} c$ will then be a homotopy equivalence. I thank W. D. Neumann for the proof of the following algebraic lemma. Let $F_{n}$ be the free abelian group of rank $n$ and $G$ a finitely generated abelian group; suppose $i_{1}$ and $i_{2}$ are epimorphisms $G \rightarrow F_{n}$;

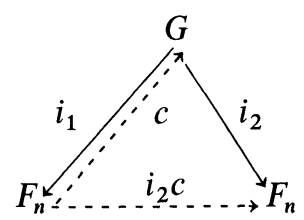

consider the problem of finding a homomorphism $c: F_{n} \rightarrow G$ such that $i_{1} c=$ identity and $i_{2} c$ is an isomorphism.

LEMMA. $c$ always exists, if we are allowed to stabilize, i.e. if we consider the diagram

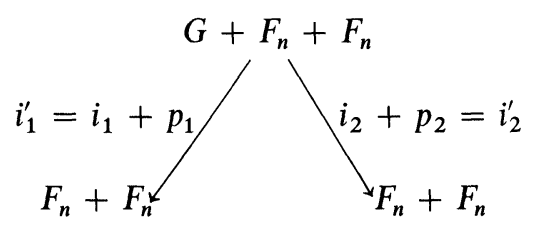

instead of (1). Here $p_{1}$ and $p_{2}$ denote the projections $F_{n}+F_{n} \rightarrow F_{n}$.

ProOF. Since $F_{n}$ is free, there exist homomorphisms $g_{1}, g_{2}: F_{n} \rightarrow G$ such 
that $i_{1} g_{1}=$ identity $=i_{2} g_{2}$; define $c: F_{n}+F_{n} \rightarrow G+F_{n}+F_{n}$ by $c(x, y)=$ $\left(g_{1} x+g_{2} y-g_{1} i_{1} g_{2} y, y, x-i_{1} g_{2} y\right)$, then $i_{2}^{\prime} c(x, y)=(x, y)$ and $i_{2}^{\prime} c: F_{n}+$ $F_{n} \rightarrow F_{n}+F_{n}$ is an isomorphism, because it is an epimorphism:

$$
i_{2}^{\prime} c\left(y-i_{1} g_{2} i_{2} g_{1} y+i_{1} g_{2} x, x-i_{2} g_{1} y\right)=(x, y) .
$$

By (a) and (c) our diagram

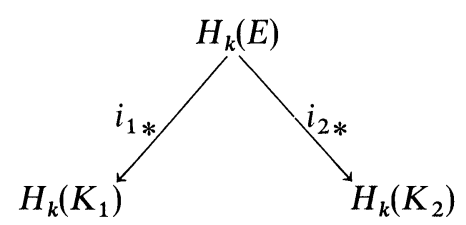

satisfies the hypothesis of the lemma; furthermore, we can suppose it has been stabilized by taking connected sums (along $E$ and $S^{k} \times S^{k}$ ) of $M^{2 k+1}=W_{1} \cup W_{2}$ with $S^{2 k+1}=S^{k} \times D^{k+1} \cup D^{k+1} \times S^{k}$ a certain number of times. We can therefore construct $c_{*}$ above and follow it up geometrically to obtain a map $c: K_{1} \rightarrow E$, such that both $i_{1} c$ and $i_{2} c$ are homotopy equivalences, which proves our assertion.

Let $V$ be a regular neighborhood of $K$ in $E$. By hypothesis, $K$ has codimension 3 and so $\partial V$ is also simply connected.

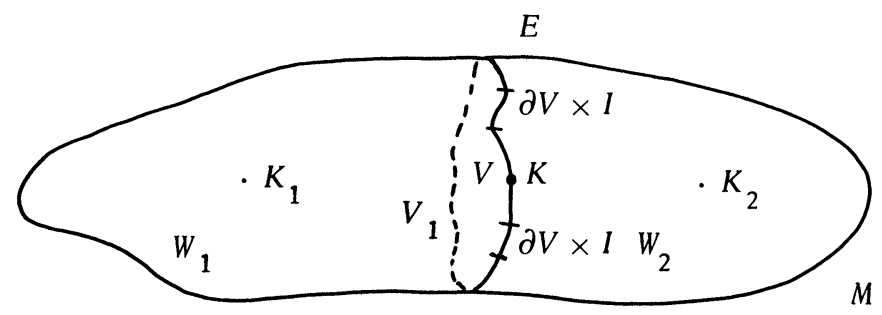

Figure 3

Take a collar neighborhood $\partial V \times I$ of $V$ in $E$ and denote by $V_{1}$ the closure of the complement of $V \cup(\partial V \times I)$ in $E$ and regard $W_{1}$ and $W_{2}$ as relative cobordisms between $V$ and $V_{1}$ (see Figure 3). $\partial V \times I$ is a product cobordism between $\partial V$ and $\partial V_{1}$ and the Assertion implies that both $W_{1}$ and $W_{2}$ are relative $h$-cobordisms.

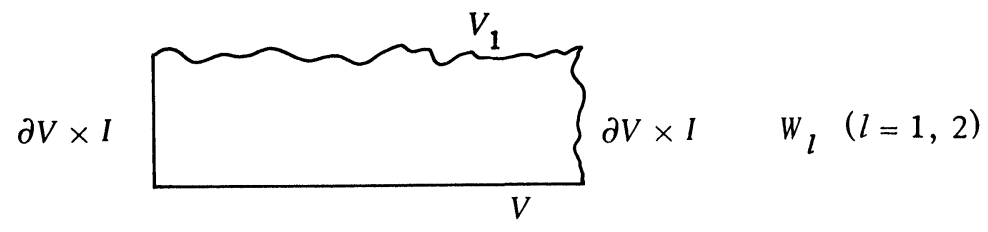

FIGURE 4 
Hence, by the relative $h$-cobordism theorem $W_{1}=V \times I=W_{2}$ and Figure 3 changes into

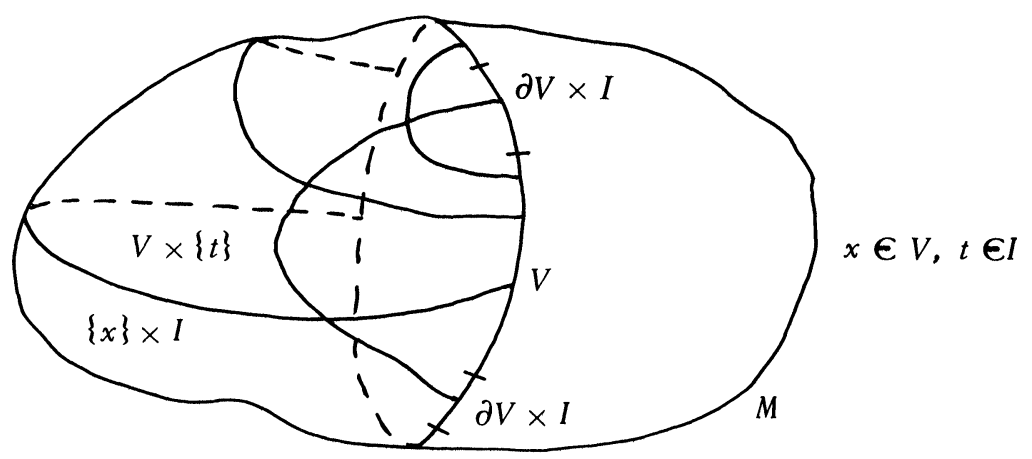

FIGURE 5

which, if we make our collar neighborhood $\partial V \times I$ smaller and smaller, changes into

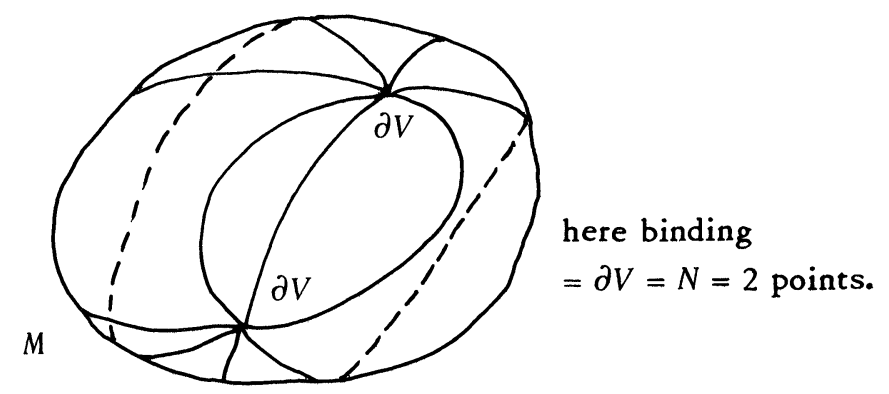

FIGURE 6

In other words, $M$ can be obtained as in Example (c) of $\$ 1$. Hence $M$ is an open book with binding diffeomorphic to $\partial V$, with simply connected pages $V$ such that $H_{i}(V, Z)=0$ for $i>[n / 2]$.

REMARK 1. For the proof of the case $n=2 k>6$ of Part 1 we split $M$ as in Chapter II of [8]: $M=W_{1} \cup W_{2}$, where $H_{i}\left(W_{l}\right)=0$ for $i>k(l=1,2)$ and the intersection forms of $W$ are $\equiv 0$ with respect to any coefficient group; the techniques of [7] then show that $W=V \times I$. Concerning Part 2 see [9] and Chapter II of [8] and for the complete details see [10]. Notice that all the above also hold in the piecewise-linear category.

REMARK 2. Recently I. Tamura independently found open book decompositions for a large subclass of simply-connected manifolds of $\tau=0$ and dimension $>6$ and N. A'Campo (to appear in Comment. Math. Helv.) proved that every simply-connected 5-manifold has an open book decomposition with binding $S^{3}$. 


\section{BIBLIOGRAPHY}

1. J. W. Alexander, A lemma on systems of knotted curves, Proc. Nat. Acad. Sci. U.S.A. 9 (1923), 93-95.

2. D. Barden, The structure of manifolds, $\mathrm{Ph} . \mathrm{D}$. Thesis, Cambridge University, 1963.

3. N. Levitt, Applications of engulfing, Ph.D. Thesis, Princeton University, Princeton, N.J., 1967

4. J. Milnor, Singular points of complex hypersurfaces, Ann. of Math. Studies, no. 61, Princeton Univ. Press, Princeton, N.J., Univ. of Tokyo Press, Tokyo, 1968. MR 39 \# 969. 5. S. Smale, On the structure of manifolds, Amer. J. Math. 84 (1962), 387-399. MR 27 \#2991.

6. C. T. C. Wall, Piecewise linear normal microbundles, Bull. Amer. Math. Soc. 71 (1965), 638-641. MR 35 \# 1038.

7. Classification problems in differential topology. IV. Thickenings, Topology 5 (1966), 73-94. MR 33 \# 734.

8. H. E. Winkelnkemper, Equators of manifolds and the action of $\Theta^{n}$, Ph.D. Thesis, Princeton University, Princeton, N.J., 1970.

9. - Manifolds as open books, Institute for Advanced Study, Princeton, N.J., 1972 (preprint).

10. - Manifolds as open books: An analogue for manifolds of Lefschetz's First theorem (to appear).

School of Mathematics, Institute for Advanced Study, Princeton, New Jersey 08540 